\title{
OTIMIZAÇÃO DA OBTENÇÃO DE BIOSSURFACTANTE POR BACILLUS SUBTILIS ATCC (9372) A PARTIR DE MELAÇO DE CANA-DE-AÇÚCAR
}

\author{
M. G. MARIM${ }^{1}$, M. V. S. LIMA¹, E. BONA ${ }^{1}$ \\ ${ }^{1}$ Universidade Tecnológica Federal do Paraná, Faculdade de Engenharia de Alimentos \\ E-mail para contato: mariliagato1@gmail.com
}

\begin{abstract}
RESUMO - Biossurfactantes são moléculas anfifílicas com aplicações industriais, que podem ser obtidas através de processo fermentativo realizado por microrganismos. Devido à crescente preocupação com o ambiente muitas pesquisas vêem sendo realizadas objetivando a obtenção de produtos e processos menos poluentes, bem como, a busca por soluções viáveis economicamente através do uso de fontes alternativas de nutrientes facilmente disponíveis e de baixo custo. Neste sentido, o presente trabalho teve como objetivo estudar o efeito do $\mathrm{pH}$; concentração inicial de inoculo; e suplementação na obtenção de biossurfactante produzido por Bacillus subtilis (ATCC 9372) empregando solução aquosa de melaço de cana-de-açúcar, clarificada, como meio de cultura. Para tanto, foi elaborado um planejamento experimental Box-Behnken para avaliar o efeito da suplementação, $\mathrm{pH}$ e quantidade de inóculo sobre a quantidade de biossurfactante e biomassa produzida durante a fermentação, conduzida sob agitação orbital de $150 \mathrm{rpm}$ por 144 horas, à $35^{\circ} \mathrm{C}$. $\mathrm{O}$ biossurfactante obtido foi recuperado através da precipitação com solvente metanol. Para quantificar a biomassa e o biossurfactante produzido em cada experimento empregou-se o método gravimétrico. Os resultados mostraram que o processo fermentativo estudado sofreu influência positiva, dentro da faixa estudada, com aumento de biomassa e biossurfactante em relação à suplementação ao nível de $1 \%, \mathrm{pH} 7 \mathrm{e}$ $1 \%$ de inóculo inicial de Bacillus subtilis.
\end{abstract}

\section{INTRODUÇÃO}

Surfactantes são compostos químicos constituídos por moléculas anfifílicas ou anfipáticas, contendo duas frações distintas: apolar (hidrofóbica) e polar (hidrofílica). Por esta razão, os surfactantes possuem a capacidade de interagir com líquidos de diferentes características como misturas apolares e polares e formar na superfície uma película ou filme, capaz de reduzir a tensão superficial na interface líquido/ar (Zana, 2005). De acordo com Sobrinho (2007), essas características conferem aos surfactantes diversas possibilidades de aplicação para uso doméstico ou industrial, tais como: detergência, emulsificação, lubrificação, espumante, molhabilidade, solubilização e dispersão de fases.

Fonseca et al. (2007) esclarece que os surfactantes mais usados atualmente são sintetizados a partir de derivados de petróleo, embora o interesse em biossurfactantes de origem microbiológica, tenha aumentado nos últimos anos devido à sua biodegradabilidade $\mathrm{e}$ 
baixa toxicidade. Porém, a produtividade e a estrutura química de um biossurfactante não dependem apenas do microrganismo utilizado, visto que, o crescimento microbiano é bastante afetado pela fonte de carbono, elementos vestigiais, temperatura, oxigênio e $\mathrm{pH}$; promovendo mudanças na estrutura química da molécula, e, conseqüentemente, alterando suas características físico-químicas e aplicação (Cara, 2009).

A literatura apresenta uma grande variedade de microrganismos, incluindo bactérias, leveduras e fungos capazes de produzir biossurfactantes com grande diversidade estrutural, porém a maioria é produzida por bactérias. Determinadas espécies do gênero Bacillus são avaliadas pela sua capacidade de produzir compostos com propriedades tensoativas, como a surfactina, produzidas por Bacilllus subtilis, caracterizada como um dos mais potentes biossurfactantes já conhecidos (Nitschke e Pastore, 2006).

Neste sentido, este trabalho teve como objetivo estudar, através de um planejamento experimental, a influência de três variáveis de processo $(\mathrm{pH}$, concentração de inoculo inicial e porcentagem de suplementação) na obtenção de biossurfactante, empregando fermentação em batelada por Bacillus subtilis (ATCC 9372) em solução aquosa de melaço de cana-de-açúcar clarificado como meio de cultura.

\section{METODOLOGIA}

Uma solução de melaço de cana-de-açúcar (100 g/L) clarificada foi utilizada como meio de cultura. A clarificação da solução de melaço foi realizada segundo metodologia descrita por Valduga (2005), e caracterizada através de análises de açúcares redutores (AR), açúcares redutores totais (ART), $\mathrm{pH}$ e teor de sólidos solúveis ( ${ }^{\circ}$ Brix), de acordo com as normas analíticas do Instituto Adolfo Lutz (1985).

A bactéria Bacillus subtilis ATCC 9372 (Bacillus atrophaeus var. subtilis) utilizada para obtenção do biossurfactante foi cedida pela empresa Clean-up Brazil Biotecnologia. A bactéria foi ativada e propagada em caldo nutriente para antes do uso na fermentação.

Para avaliar a influencia das variáveis de processo: $\mathrm{pH}$, concentração inicial de inóculo e suplementação (extrato de levedura e peptona) na produção do biossurfactatnte e da biomassa empregou-se a técnica de planejamento experimental Box-Behnken para estes três fatores de estudo, utilizando dois níveis, superior $(+1)$ e inferior $(-1)$ e um ponto central $(0)$, para cada fator. Os níveis reais e codificados estão apresentados na Tabela 1. Todos os ensaios do planejamento foram realizados aleatoriamente. As respostas avaliadas (variáveis dependentes) foram aumento de biomassa $(\mathrm{g} / \mathrm{L})$ e biossurfactante $(\mathrm{g} / \mathrm{L})$.

Tabela 1 - Níveis reais e codificados das variáveis estudadas no planejamento

\begin{tabular}{lcccc}
\hline & \multirow{2}{*}{ VARIÁVEIS } & \multicolumn{3}{c}{ Níveis reais e codificados } \\
\cline { 3 - 5 } & & 5,0 & 0 & 1 \\
\hline X1 & pH da reação & 6,0 & 7,0 \\
X2 & Inóculo $(\%$ V/V) & 1,0 & 1,5 & 2,0 \\
X3 & Suplementação $(\% \mathrm{~m} / \mathrm{V})$ & 0 & 0,5 & 1,0 \\
\hline
\end{tabular}


Toda análise estatística foi realizada utilizando o software STATISTICA 7.1 (Statsoft Inc. 2325 East 13th Street, Tulsa, OK, 74104, USA). Para avaliar o efeito das variáveis independentes sobre as variáveis dependentes (respostas) a metodologia de superfície de resposta foi aplicada; e a análise de variância foi utilizada para avaliar a adequação dos modelos que foram ajustados a partir da Equação 1.

$$
y\left(X_{1}, X_{2}, X_{3}\right)=\beta_{0}+\beta_{1} X_{1}+\beta_{2} X_{2}+\beta_{3} X_{3}+\beta_{11} X_{1}^{2}+\beta_{22} X_{2}^{2}+\beta_{33} X_{3}^{2}+\beta_{12} X_{1} X_{2}+\beta_{23} X_{2} X_{3}+\beta_{123} X_{1} X_{2} X_{3}
$$

Onde y é a resposta prevista, $\beta_{0}$ é uma constante, $\beta_{1}, \beta_{2}, \beta_{11}, \beta_{22}$ e $\beta_{12}$ são os coeficientes da regressão e $X_{1}, X_{2} X_{3}$ são os níveis das variáveis independentes.

Os experimentos foram realizadas em frascos Erlenmeyer de $250 \mathrm{~mL}$ de capacidade, contendo $100 \mathrm{~mL}$ de meio de cultura composto pela solução aquosa de melaço clarificado na concentração $100 \mathrm{~g} / \mathrm{L}$. O meio de cultura foi inoculado, com a solução de inoculo contendo $6,7 \times 10^{8} \mathrm{UFC} / \mathrm{mL}$ de Bacillus subtilis, ajustado o $\mathrm{pH}$ e adicionado os suplementos (extrato de levedura e peptona) de acordo com o planejamento experimental proposto. Para determinação de biomassa inicial (massa seca) foi retirada uma amostra de $10 \mathrm{~mL}$ e após os frascos foram mantidos fechados sob agitação orbital de $150 \mathrm{rpm}$ durante 144 horas, à $35^{\circ} \mathrm{C}$ em Banho Termostático Agitado MOD-DI - $950 \mathrm{M}$.

A determinação de biomassa seca foi realizada no início e ao término da fermentação. Além da quantificação da biomassa e do biossurfactante produzido também foram feitas leituras de $\mathrm{pH}$ ao témino da fermentação.

O biossurfactante produzido durante as fermentações foi recuperado do caldo fermentado segundo a metodologia sugerida por Sobrinho (2007), onde o caldo fermentado foi centrifugado (4500 rpm) por 20 minutos, então o sobrenadante teve seu $\mathrm{pH}$ ajustado para 2 com solução de $\mathrm{HCl} 6,0 \mathrm{~mol} / \mathrm{L}$ e precipitado com 2 volumes de metanol. Após repouso por 24 horas à $0^{\circ} \mathrm{C}$, foi centrifugado $(4000 \mathrm{rpm})$ por 30 minutos, e o precipitado foi lavado duas vezes com metanol gelado e então seco em estufa a $37^{\circ} \mathrm{C}$ por 48 horas, o biossurfactante seco foi pesado; e calculou-se a quantidade de biossurfactante obtido para cada experimento em $\mathrm{g} / \mathrm{L}$.

\section{RESULTADOS}

Os resultados das determinações analíticas de ${ }^{\circ}$ Brix; AR; ART do caldo foram de 8 ${ }^{\circ}$ Brix, $5,5 \mathrm{~g} / \mathrm{L}, 23,21 \mathrm{~g} / \mathrm{L}$, respectivamente, confirmando concentrações adequadas para suprir as necessidades dos microrganismos durante o processo fermentativo e contribuindo com o aumento de biomassa e produção do biossurfactante.

Ao analisar a tabela 2, observa-se que o $\mathrm{pH}$ diminuiu em todos os experimentos mantendo-se numa faixa de $\mathrm{pH}$ final de 4 a 4,5, o que também foi observado por Ciapina (2008) e Pirog et al. (2004). Essa acidificação é causada devido ao melaço de cana-de-açúcar propiciar a formação de outros metabólitos pelo microrganismo Bacillus subtilis, como ácidos orgânicos, que alteraram o $\mathrm{pH}$ do meio. 
A Tabela 2 também apresenta a matriz de ensaio com os valores reais (entre parênteses) e codificados das variáveis estudadas, bem como, as respostas obtidas para o aumento da biomassa e biossurfactante, e $\mathrm{pH}$ final.

Tabela 2 - Respostas da matriz de planejamento Box-Behnken

\begin{tabular}{ccccccc}
\hline Ensaio & $\mathbf{x}_{\mathbf{1}}{ }^{\mathbf{a}}$ & $\mathbf{x}_{\mathbf{2}}{ }^{\mathbf{b}}$ & $\mathbf{x}_{\mathbf{3}}{ }^{\mathbf{c}}$ & $\mathbf{p H}$ final & $\begin{array}{c}\text { Aumento de } \\
\text { Biomassa } \\
\mathbf{( g / L )}\end{array}$ & $\begin{array}{c}\text { Biossurfactante } \\
\mathbf{( g / L )}\end{array}$ \\
\hline $\mathbf{1}$ & $-1(5,0)$ & $-1(1,0)$ & $0(0,5)$ & 4,4 & 1,09 & 1,08 \\
$\mathbf{2}$ & $+1(7,0)$ & $-1(1,0)$ & $0(0,5)$ & 4,16 & 1,21 & 1,20 \\
$\mathbf{3}$ & $-1(5,0)$ & $+1(2,0)$ & $0(0,5)$ & 4,19 & 1,31 & 1,39 \\
$\mathbf{4}$ & $+1(7,0)$ & $+1(2,0)$ & $0(0,5)$ & 4,2 & 1,12 & 1,32 \\
$\mathbf{5}$ & $-1(5,0)$ & $0(1,5)$ & $-1(0,0)$ & 4,15 & 1,21 & 1,55 \\
$\mathbf{6}$ & $+1(7,0)$ & $0(1,5)$ & $-1(0,0)$ & 4,37 & 1,29 & 2,07 \\
$\mathbf{7}$ & $-1(5,0)$ & $0(1,5)$ & $+1(1,0)$ & 4,34 & 2,19 & 3,54 \\
$\mathbf{8}$ & $+1(7,0)$ & $0(1,5)$ & $+1(1,0)$ & 4,46 & 2,11 & 3,65 \\
$\mathbf{9}$ & $0(6,0)$ & $-1(1,0)$ & $-1(0,0)$ & 4,41 & 1,37 & 1,27 \\
$\mathbf{1 0}$ & $0(6,0)$ & $+1(2,0)$ & $-1(0,0)$ & 4,75 & 1,08 & 1,17 \\
$\mathbf{1 1}$ & $0(6,0)$ & $-1(1,0)$ & $+1(1,0)$ & 4,47 & 2,26 & 2,78 \\
$\mathbf{1 2}$ & $0(6,0)$ & $+1(2,0)$ & $+1(1,0)$ & 4,29 & 1,71 & 2,93 \\
$\mathbf{1 3}$ & $0(6,0)$ & $0(1,5)$ & $0(0,5)$ & 4,28 & 1,41 & 1,77 \\
$\mathbf{1 4}$ & $0(6,0)$ & $0(1,5)$ & $0(0,5)$ & 4,22 & 1,41 & 1,77 \\
$\mathbf{1 5}$ & $0(6,0)$ & $0(1,5)$ & $0(0,5)$ & 4,3 & 1,36 & 1,77 \\
\hline${ }^{\mathrm{a}} \mathrm{pH}$ da reação. & & & & & \\
${ }_{\mathrm{b}}$ Inóculo $(\% \mathrm{~V} / \mathrm{V})$. & & & &
\end{tabular}

De acordo com os coeficientes de regressão para a resposta biomassa $(\mathrm{g} / \mathrm{L})$ as variáveis apresentaram efeitos estatisticamente significativos $(\mathrm{p} \leq 0,05)$, com exceção do $\mathrm{pH}$. Porém, apenas a suplementação teve efeito positivo sobre a resposta, ou seja, maiores valores de biomassa foram observados quando a suplementação estava no nível superior $(+1)$.

Considerando-se os parâmetros significativos $(\mathrm{p} \leq 0,05)$ obteve-se a Equação 1, que representa o modelo quadrático da biomassa em função das variáveis estudadas. Os parâmetros não significativos foram incorporados aos resíduos para o cálculo da análise de variância (ANOVA). O $\mathrm{F}_{\text {calculado }}$ da regressão apresentou-se significativo $(0,001088)$, sendo maior que o $\mathrm{F}_{\text {tabelado, }}$ e o percentual de variação explicada pelo modelo foi adequado $\left(\mathrm{R}^{2} \approx\right.$ $87 \%$ ), podendo assim, concluir que o modelo (Equação 2) ajustou-se bem aos dados experimentais.

Biomassa $(g / L)=1389,81-7,14 X_{1}-89,29 X_{2}+417,14 X_{3}-148,83 X_{2}^{2}+364,02 X_{3}^{2}-76,43 X_{12}-65,00 X_{23}$

Ao analisar os resultados da Tabela 2 referente às respostas de Biossurfactante $(\mathrm{g} / \mathrm{L})$ juntamente com os coeficientes de regressão, nota-se que o efeito da suplementação, $\mathrm{pH}$ e interação inóculo com suplementação $\left(\mathrm{x}_{2} \cdot \mathrm{x}_{3}\right)$ foram positivos, afirmando que quando usados no nível superior aumentam a produção de biossurfactante. Assim, a máxima produtividade de biossurfactante foi constatada com 1\% (+1) de suplementação, pH ajustado para $7(+1)$ e $1 \%$ (-1) de inóculo de Bacillus subtilis, conforme mostra a Figura 1.

Figura 1 - Curva de contorno para o biossurfactante com concentração de inóculo fixa no ponto central. 

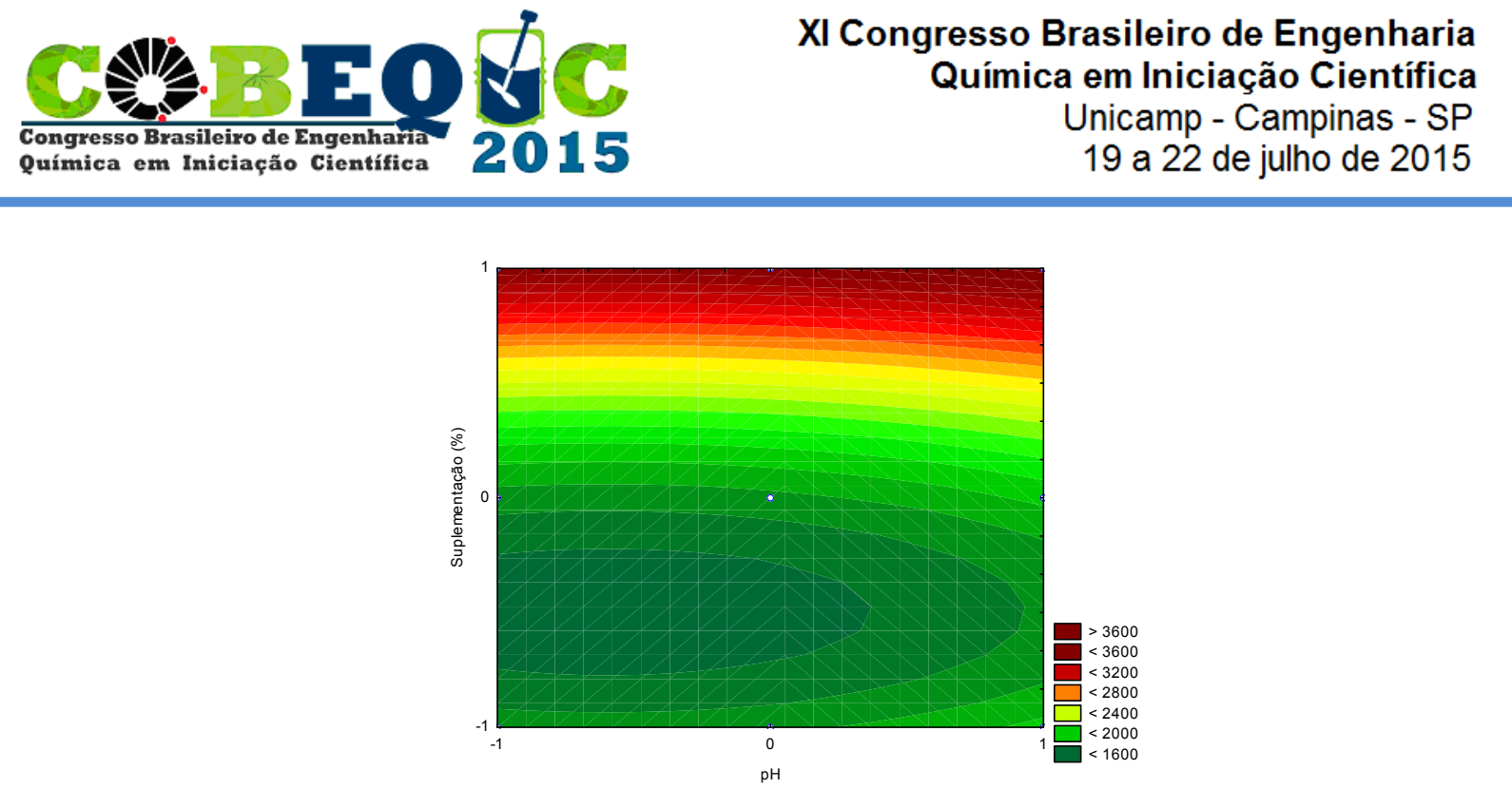

Considerando os parâmetros significativos $(p \leq 0,05)$ obteve-se a Equação 3 , que representa o modelo quadrático de biossurfactante em função das variáveis estudadas. Os parâmetros não significativos foram incorporados aos resíduos para o cálculo da análise de variância (ANOVA). O $\mathrm{F}_{\text {calculado }}$ da regressão apresentou-se significativo (0,000075), sendo maior que o $\mathrm{F}_{\text {tabelado, }}$ e o percentual de variação explicada pelo modelo foi adequado $\left(\mathrm{R}^{2} \approx\right.$ 98\%), podendo assim, concluir que o modelo ajustou-se bem aos dados experimentais.

Biossurfactante $(m g / L)=1770,71+85,00 X_{1}+60,54 X_{2}+860,89 X_{3}+71,25 X_{1}^{2}-592,68 X_{2}^{2}+860,89 X_{3}^{2}-47,14 X_{12}-1$

Torna-se interessante ressaltar que, as melhores condições para o desenvolvimento do microrganismo e produção de biossurfactane, foram as que tiveram a adição de suplementação e ficaram na faixa do $\mathrm{pH}$ neutro ou $\mathrm{pH}$ 7. Observa-se que a concentração de biossurfactante variou de 3,65 a 1,08 g/L (ensaios 8 e 1). Essa faixa de resultados referente ao biossurfactante obtido pode ser considerada adequada, visto que Penna el. al (2014), avaliou a produção de biossurfactantes a partir do cultivo de Bacillus atrophaeus ATCC 9372 e Bacillus subtilis W1012 (GFP uv) em meio TSB (Tryptone Soy Broth) e alcançou produção de $0,40 \mathrm{~g} / \mathrm{L}$ e $0,50 \mathrm{~g} / \mathrm{L}$ respectivamente. Pode-se notar que nas condições estudadas o processo fermentativo proporcionou alta concentração de biossurfactante quando comparado ao trabalho de Penna et. al. para o mesmo microrganismo. -Em relação ao meio composto por melaço suplementado com extrato de levedura e peptona, este teve influência positiva e foi capaz de promover o crescimento do microrganismo e produção do biossurfactante.

\section{CONCLUSÃO}

Os resultados sugerem viabilidade técnica para produção de biossurfactante a partir de resíduos agroindustriais, como o melaço de cana de açúcar, e do Bacillus subtilis (ATCC 9372) como biocatalisador do processo. Pode-se concluir que o processo fermentativo estudado sofreu influência positiva em relação à suplementação com peptona e extrato de levedura ao nível de 1\%(+1), pH $7(+1)$ e 1\% (-1) de inóculo inicial de Bacillus subtilis, dentro da faixa estudada. Vale salientar que o fator suplementação favoreceu tanto o aumento da biomassa quanto a produção de biossurfactante.

\section{5- REFERÊNCIAS}


CARA, D. V. C. Produção de biossurfatante por flavobacterium sp. a partir de óleo de soja residual e fertilizante comercial. 2009. $71 \mathrm{f}$. Tese (Mestrado) - Universidade Federal do Rio de Janeiro, Escola de Química, Rio de Janeiro, 2009.

CIAPINA, E. M. P. Produção de Biossurfactante por Rhodococcus erythropolis em Biorreator de Bancada e Avaliação do seu Efeito na Biodegradação de Borra Oleosa da Indústria do Petróleo. Tese (Doutorado em Tecnologia de Processos Químicos e Bioquímicos) - Universidade Federal do Rio de Janeiro - UFRJ, Instituto de Pós Graduação em Tecnologia de Processos Químicos e Bioquímicos. Rio de Janeiro, 2008.

FONSECA, R. R.et al. Optimizing Carbon/ Nitrogen Ratio for Biosurfactant Production by a Bacillus subtilis Strain. Applied Biochemistry and Biotechnology, Rio de Janeiro, v. 136- 140, p. 471- 486, 2007.

INSTITUTO ADOLFO LUTZ. Normas Analíticas do Instituto Adolfo Lutz. v. 1: Métodos químicos e físicos para análise de alimentos, 3 ed. São Paulo: IMESP,p. 157, 1985.

NITSCHKE, M.; PASTORE, G. M. Production and properties of a surfactant obtained from Bacillus subtilis grown on cassava wastewater. Bioresource Technology, v. 97, p. 336-341, 2006.

PENNA, T. C. V. et al. Avaliação da Produção de biossurfactantes a partir do cultivo de Bacillus atrophaeus ATCC 9372 e Bacillus subtilis W1012 (GFP uv) em meio TSB (Tryptone Soya Broth). Departamento de Tecnologia Bioquímico-Farmacêutica, Faculdade de Ciências Farmacêuticas, Universidade de São Paulo, 2014.

PIROG, T. P.; SHEVCHUK, T.A.; VOLOSHINA, I. N. AND KARPENKO, E. V. Production of surfactants by Rhodococcuserythropolisstrain EK-1, grown on hydrophilic and hydrophobic substrates. Applied Biochemistry and Microbiology, v. 40, p. 544-550, 2004.

SOBRINHO, H. B. S. Utilização de resíduos industriais como substratos de baixo custo para a produção de biossurfactante por Candida sphaerica. 2007. 98 f. Dissertação (Mestrado em Desenvolvimento de Processos Ambientais) - Universidade Católica de Pernambuco, Recife, 2007.

VALDUGA, E. Bioprodução de compostos voláteis e carotenoides por Sporodiobolus salmonicolor CBS 2636. 2005. 189 f. Tese (Doutorado) - Universidade Federal de Santa Catarina, Florianópolis. 2005.

ZANA, R. Dynamics of surfactant self-assemblies: micelles, micro emulsions, vesicles, and lyotropic phases. (Surfactant science series; v. 125) New York: CRC Press, 2005. 539. Disponível em: < http://migre.me/kmIz6 >Acesso em: 22 mai.2014 